\title{
Nutrition Therapy in Non-intubated Patients with Acute Respiratory Failure
}

\author{
Nadine Sbaih ${ }^{1} \cdot$ Kelly Hawthorne ${ }^{2} \cdot$ Jennifer Lutes ${ }^{3} \cdot$ Rodrigo Cavallazzi $^{3,4}$
}

Accepted: 29 June 2021 / Published online: 31 August 2021

This is a U.S. government work and not under copyright protection in the U.S.; foreign copyright protection may apply 2021

\begin{abstract}
Purpose of Review A challenging aspect of the care for patients with acute respiratory failure is their nutrition management. This manuscript consists of a literature review on nutrition therapy in non-intubated patients with acute respiratory failure receiving high-flow nasal cannula oxygenation or non-invasive positive pressure ventilation.

Recent Findings Studies show that non-intubated patients with acute respiratory failure either on non-invasive ventilation or high-flow nasal cannula are largely underfed in the initial phase of their hospitalization. Although data is limited, the available evidence suggests the feasibility of initiating oral diet in the majority of these patients in the early phase.

Summary Initial evaluation includes mental status evaluation, the Yale swallowing screening protocol, and an assessment of severity of illness. The goal should be to initiate oral diet within $24 \mathrm{~h}$. If patient cannot initiate oral diet, the reason for not initiating oral diet should dictate the next step. For instance, if the reason is failure of the swallow screening, further evaluation with fiberoptic endoscopy is warranted. The inability to provide oral diet for a patient in respiratory distress may a harbinger of failure of non-invasive oxygen therapy and should prompt consideration for endotracheal intubation. We suggest placement of a small-bore feeding tube for enteral nutrition if patient is unable receive oral diet after $48 \mathrm{~h}$.

Conclusions The nutrition management of these patients is better provided by a multidisciplinary team in a protocolized manner.
\end{abstract}

Keywords Acute respiratory failure $\cdot$ COVID- $19 \cdot$ NIV $\cdot$ BIPAP $\cdot$ Nutrition

\section{Introduction}

A challenging aspect of the care for patients in acute respiratory failure is their nutrition management. Several features of nutrition have been studied in patients in acute respiratory failure on invasive mechanical ventilation. These include trophic

This article is part of the Topical Collection on Gastroenterology, Critical Care, and Lifestyle Medicine

Rodrigo Cavallazzi

r0cava01@louisville.edu

1 Department of Medicine, University of Louisville, Louisville, KY, USA

2 Nutrition Services, University of Louisville Hospital, Louisville, KY, USA

3 Speech-Language Pathology, University of Louisville Hospital, Louisville, KY, USA

4 Division of Pulmonary, Critical Care Medicine, and Sleep Disorders, University of Louisville, Louisville, KY, USA vs full enteral feeding [1], and early vs late enteral nutrition of enteral feeding [2]. However, sparse data is available on nutrition in non-intubated patients in acute respiratory failure. Nonetheless, nutrition management of the latter patients remains clinically important and was brought to the spotlight during the coronavirus disease 2019 (Covid-19) pandemic. This manuscript consists of a literature review on nutrition therapy in non-intubated patients with acute respiratory failure. We focus on patients receiving high-flow nasal cannula oxygenation or non-invasive positive pressure ventilation, the two commonly used forms of non-invasive oxygen delivery in patients in severe acute respiratory failure.

\section{Types of Acute Respiratory Failure}

Acute respiratory failure is defined as failure of oxygenation, ventilation, or both. From a pathophysiological standpoint, respiratory failure is divided into type I (hypoxemic) and type II (hypercapnic) [3, 4]. Type I acute respiratory failure is a result of gas exchange failure, originating at the level of pulmonary 
intrinsic structure. The presence of $\mathrm{PaO} 2 \leq 60 \mathrm{mmHg}$ indicates oxygenation failure. The different mechanisms of type I acute respiratory failure include ventilation-perfusion mismatch, alveolar hypoventilation, diffusion impairment, right to left shunting of blood, and low fraction of inspired oxygen. The most common mechanism of hypoxemic respiratory failure is ventilationperfusion mismatch, in which there is a decrease in ventilation in relation to perfusion. The extreme form of ventilation-perfusion mismatch is shunt, which is characterized by no ventilation in the setting of perfusion. Impaired diffusion leads to hypoxemia by making it difficult for the oxygen to diffuse across the alveolocapillary membrane [3]. In diffusion limitation due to inflammation or fibrosis, it is uncommon to also see hypercapnia. This is because carbon dioxide is about 20 times more soluble than oxygen; therefore, carbon dioxide is usually not limited by diffusion impairment [5].

Type II acute respiratory failure occurs when there is ventilatory failure. This results in hypercapnia, which is defined as having a PaCO2 $>45 \mathrm{mmHg}$. Etiologies that cause hypercapnic respiratory failure include decreased respiratory drive, chest wall mechanical deficits, increase in dead space, disease of respiratory muscles, or increased carbon dioxide production [3]. In a patient with hypoxemia, the analysis of the alveolar-arterial oxygen gradient is valuable to assess what type of respiratory failure the patient has. In pure hypercapnic respiratory failure, there should not be a large gap between the alveolar and arterial oxygen tension, and the alveolar-arterial oxygen gradient is normal because the intrinsic function of the lungs is intact. Conversely, in patients with hypoxemic respiratory failure, there is a wide gap between the alveolar and arterial oxygen tension, which is secondary to a pulmonary structural abnormality leading to reduced efficiency of oxygen transfer [4]. Table 1 provides a summary of the types and causes of acute respiratory failure based on comprehensive reviews of the literature [4-6].

\section{Non-invasive Ventilation and High-Flow Nasal Cannula}

Non-invasive ventilation can be applied with different modes, but the most common are bilevel positive pressure ventilation and continuous positive airway pressure. Three decades ago, there was a report showing the feasibility of non-invasive intermittent positive pressure ventilation delivered via face mask for patients with acute respiratory failure [7, 8]. Subsequent studies provided more detail on the physiology of this mode of ventilation, which was then widely incorporated into clinical practice. There is currently data showing a benefit of non-invasive ventilation with bilevel positive pressure in patients with COPD presenting with hypercapnic respiratory failure. Additionally, there is a benefit of non-invasive ventilation with either bilevel positive pressure or continuous positive airways pressure in patients with acute respiratory failure secondary to cardiogenic pulmonary edema. In these 2 groups of patients, the use of non-invasive ventilation leads to a reduction in the need for endotracheal intubation and mortality $[9,10]$. Non-invasive ventilation has also been used in other settings of acute respiratory failure although here the data is neither as robust nor as compelling.

High-flow nasal cannula oxygenation device is a tool that has been increasingly used for the treatment of acute hypoxic respiratory failure. The device contains air-oxygen blender, active humidifier, heated inspiratory circuit, and a nasal cannula. The FiO2 and the flow can be independently adjusted. A distinctive feature of the device is the ability of provide oxygen flow of up to $60 \mathrm{~L} / \mathrm{min}$. Physiological effects of the device include a decrease in the anatomical dead space, an increase in positive airway pressure, and a flow that, if properly adjusted, can match the patient's demand [11].

\section{Energy Expenditure in Patients with Acute Respiratory Failure}

Indirect calorimetry is considered the most reliable method for assessment of energy expenditure. The metabolism of nutrients on the presence of oxygen generates carbon dioxide and water. In steady state, the oxygen consumption is equivalent to carbon dioxide excreted [12]. This is exemplified by the metabolism of glucose:

$$
\mathrm{C} 6 \mathrm{H} 12 \mathrm{O} 6+6 \mathrm{O} 2 \rightarrow 6 \mathrm{H} 2 \mathrm{O}+6 \mathrm{CO} 2+\text { energy }
$$

In indirect calorimetry, the amount of oxygen consumed $\left(\mathrm{VO}_{2}\right)$ and carbon dioxide excreted $\left(\mathrm{VCO}_{2}\right)$ are measured and

Table 1 Causes of acute respiratory failure according to pathophysiological mechanism

\begin{tabular}{|c|c|}
\hline Type 1 (hypoxic) & Type 2 (hypercapnic) \\
\hline $\begin{array}{l}\text { Ventilation/perfusion mismatch: obstructive pulmonary diseases } \\
\text { (asthma, chronic obstructive pulmonary disease, bronchiectasis, } \\
\text { cystic fibrosis), interstitial lung disease, pulmonary embolism, } \\
\text { pneumonia, pulmonary edema, acute respiratory distress syndrome }\end{array}$ & $\begin{array}{l}\text { Impaired central drive due to drug overdose, brainstem damage, or central } \\
\text { alveolar hypoventilation }\end{array}$ \\
\hline $\begin{array}{l}\text { Right to left shunt (patent foramen ovale, atrial septal defect, } \\
\text { ventricular septal defect, pulmonary arteriovenous malformation, } \\
\text { hepatopulmonary syndrome) }\end{array}$ & $\begin{array}{l}\text { Chest wall mechanical deficits such as kyphoscoliosis, thoracoplasty, and } \\
\text { fibrothorax) }\end{array}$ \\
\hline Diffusion impairment (emphysema, interstitial lung disease) & $\begin{array}{l}\text { Neuromuscular and myopathies affecting respiratory muscles (myasthenia } \\
\text { gravis, Guillain-Barré syndrome, amyotrophic lateral sclerosis) }\end{array}$ \\
\hline
\end{tabular}


allow for the calculation of the resting energy expenditure based on an abbreviation of the modified Weir equation [13]:
Using the estimated requirements as the standard, $80 \%$ of the energy requirements and $80 \%$ of the protein requirements

Energy expenditure $(\mathrm{Kcal} /$ day $):\left(\left[\mathrm{VO}_{2} \times 3.941\right]+\left[\mathrm{VCO}_{2} \times 1.11\right]\right) \times 1440$

There is a contentious debate as to whether indirect calorimetry should be routinely implemented in clinical practice $[14,15]$. While some experts advocate for the routine use of indirect calorimetry in critically ill patients [14], others suggest its use for situations in which weight-based formulas are likely to be inaccurate such as anasarca, severe obesity, or the recovery phase of critical illness [16]. There is, however, consensus that indirect calorimetry remains the gold standard for measuring energy expenditure and it allows for a better understanding of metabolic processes taking place in patients in acute respiratory failure. Research studies using indirect calorimetry have shown that the metabolic needs of these patients can vary during hospitalization and also differ according to the etiology of respiratory failure [14]. For example, in patients admitted to the hospital with COPD exacerbation, resting energy expenditure is initially elevated but it starts to decrease to baseline levels after 3 days. The initial elevated resting energy expenditure is not matched by dietary intake in these patients, which is impaired [17]. On the other hand, in patients with Covid-19, there is a hypermetabolism that exceedes Harris-Bennedict equation prediction after the first week. This hypermetaboism increases throughout the hospitalization with a peak in the third week [18•].

Other methods for determining energy requirements include predictive equations and simplistic weight-based equations. The 2016 American Society for Parenteral and Enteral Nutrition guidelines point out that predictive equations do not have good accuracy and suggest the use of weightbased Eq. (25-30 kcal/kg/day) for determination of energy requirements in the absence of indirect calorimetry [15]. A more recent statement for patients with Covid-19 requiring nutritional therapy recommend early enteral nutrition initiation (within 24-36 h of ICU admission), but a lower energy goal of $15-20 \mathrm{kcal} / \mathrm{kg}$ of actual body weight [19]. The latter recommendation is more in line with what we have been practicing in patients with acute respiratory failure requiring nutritional support, but it does not necessarily apply to all patients as those with malnutrition may need higher caloric provision with careful monitoring for refeeding syndrome.

\section{The Current Status of Nutrition Practice for Patients in Acute Respiratory Failure}

In a study that included 37 patients receiving non-invasive ventilation for acute respiratory failure, energy and protein consumed by the patients were measured by the nursing staff. were not met by $78 \%$ and $75 \%$ of the patients, respectively [20]. In a study that included patients on non-invasive ventilation in 20 French ICUs, their nutritional management was recorded in the first $48 \mathrm{~h}$. Of 1075 patients, 622 patients $(57.9 \%)$ received no nutrition, 351 (33\%) received oral nutrition only, 74 (7\%) received parenteral nutrition only, and 28 (3\%) received enteral nutrition [21]. In a study of 50 adult patients on high-flow nasal cannula, 39 (78\%) were started on oral nutrition whereas $11(22 \%)$ were kept nil per os [22]. In another study of 40 adult critically ill patients who were treated with high-flow nasal cannula during the first 2 days of admission to the ICU, they received on average only $449.5 \mathrm{kcal} /$ day and $19.25 \mathrm{~g} /$ day protein [23•].

Patients with acute respiratory failure represent a heterogenous population. Therefore, it is conceivable that nutrition practice varies within this patient population depending on factors such as severity of illness or the etiology of acute respiratory failure. But the studies show that on aggregate non-intubated patients in acute respiratory failure either on non-invasive ventilation or high-flow nasal cannula are largely underfed in the initial phase of their hospitalization.

\section{Assessing the Nourishment Status Upon Admission}

Important changes in the nutrition approach to critically ill patients have taken place during the past decade. The intensive provision of full caloric requirements in the initial phase of critical illness has been replaced by a strategy consisting of initial trophic feeding and slow advancement to caloric goals. Early initiation of nutrition and high protein feeding remain important, but the caloric goal is set at 70 to $80 \%$ of the estimated caloric requirement in the initial 5 to 7 days of hospital admission [16]. The bases for this new approach were randomized, multicenter clinical trials in critically ill patients that showed that underfeeding, as compared to full feeding, did not worsen outcomes [24, 25] and could improve gastrointestinal tolerance [24]. It is important to note that these studies were conducted in patients receiving invasive mechanical ventilation, and the extrapolation of their results to non-intubated patients may not hold true. Perhaps equally important is the observation that in these trials patients had an average body mass index of $29-30 \mathrm{~kg} / \mathrm{m}^{2}$, implying that on aggregate patients were on the edge between overweight and obesity classes. These body mass index figures do not mean patients were adequately nourished since it has been shown 
that a substantial proportion of patients with malnutrition are overweight or obese [26]. But they bring the question as to whether patients enrolled in clinical trials are fully representative of the population seen in clinical practice. A comprehensive review of the literature noted that approximately one-third of hospitalized patients in the USA or Europe are malnourished or at nutritional risk. Malnourishment in these patients is associated prolonged length of stay and increased mortality [27]. It is, therefore, critical to recognize these patients as they may need personalized nutrition therapy.

Assessment of nourishment can be accomplished by history taking and physical examination or with the use of a nutrition score, but a precise definition of malnutrition has always been challenging. In order to standardize the diagnosis of malnutrition, a consensus statement from the Academy of Nutrition and Dietetics and American Society for Parenteral and Enteral Nutrition recommended that the presence of at least 2 of the following 6 features needs to be present for the diagnosis: insufficient energy intake, weight loss, loss of muscle mass, loss of subcutaneous fat, fluid accumulation, and diminished functional status [28].

There are several nutrition scores available that can be used to assess nutritional risk. Two main components should be included in a nutritional risk assessment: nutritional status and severity of illness. Assessment of the nutritional status component provides insight into the nutritional risk related to chronic diseases, whereas the severity of illness component helps to gauge the nutritional risk related to the acute illness. The integration of both components should identify patients more likely to benefit from nutritional support [29].

The American clinical nutrition guidelines suggest the use of NUTRIC score or nutritional risk screening, both of which assess nutritional status and severity of illness [15]. The NUTRIC score is based on a model that contemplates inflammation, starvation, and outcome. Variables of the NUTRIC score include age, Acute Physiology and Chronic Health Evaluation II (APACHE II) system, Sequential Organ Failure Assessment (SOFA) score, number of co-morbidities, days from hospital to ICU admission, and interleukin-6. In the original validation study that included 597 critically ill patients, it was shown that higher NUTRIC scores were associated with higher 28 -day mortality and duration of mechanical ventilation. Additionally, for those who stayed in the ICU for 3 days or longer, a higher NUTRIC score identified those who are more likely to benefit from increasing caloric intake [30].

In the nutritional risk screening, undernutrition is graded according to weight loss, body mass index, food intake, and age, whereas severity of disease is graded according to the estimated nutritional requirement deriving from the disease [31]. In a prospective study of 292 patients, those at risk of malnutrition as assessed by nutritional risk screening had longer length of hospital stay [26].

Malnutrition screening tool is another method to identify patients who are malnourished or at risk of becoming malnourished. It includes questions regarding weight loss and appetite. In a study that included 408 patients, both the agreement among evaluators using the tool and the accuracy of the tool to indentify malnutrition were high. Patients at risk of manutrition as determined by the malnutrition screening tool had longer length of stay [32]. Table 2 summarizes the 3 systems discussed above to screen for malnutrition or risk of malnutrition.

It has become increasingly recognized that serum albumin and pre-albumin are markers of inflammation, but not of malnutrition itself. Declined levels of these markers can indicate the presence of inflammation and thus increased nutrition risk and worse outcomes, but they do not necessarily represent decreased muscle mass [33]. Imaging studies such as portable ultrasound and computed tomography are emerging as important modalities to assess muscle mass and may have a more pronounced role in the nutritional assessment of critically ill patients in the near future [34].

\section{Nutrition via Oral Route in Patients with High-Flow Nasal Cannula}

Despite beneficial physiological effects in acute respiratory failure, high-flow nasal cannula can also have an effect on the swallowing. In healthy adults, studies have provided conflicting results regarding this effect. For instance, in a

Table 2 Summary of three tools to screen for malnutrition or risk of malnutrition

\begin{tabular}{ll}
\hline Nutrition screening tools & Screening parameters/scoring criteria \\
\hline Malnutrition Screening Tool (MST) [32] & Unintentional weight loss within 6 months, appetite; at-risk score $\geq 2$ \\
Nutritional Risk Screening-2002 (NRS 2002) [31] & Age, unintentional weight loss within 3 months, nutritional intake, body mass \\
& index, disease severity; at-risk score $\geq 3$ \\
Nutrition Risk in the Critically Ill (NUTRIC Score) [30] & Age, APACHE II Score, SOFA Score, number of comorbidities, days in \\
hospital to ICU admit, IL-6 (optional) & With IL-6: 6-10 is high malnutrition risk, 0-5 is low malnutrition risk \\
& Without IL-6: 5-9 is high malnutrition risk, 0-4 is low malnutrition risk
\end{tabular}

APACHE Acute Physiology and Chronic Health Evaluation, SOFA Sequential Organ Failure Assessment, IL interleukin 
study of 29 healthy adults, swallowing was evaluated via videofluoroscopic swallow study at flow rates of 10, 20, 30, 40, 50, and $60 \mathrm{~L} / \mathrm{min}$. Aspiration was not visualized, and increasing airflow was associated with increasing laryngeal vestibule closure. Although no association was objectively observed between penetration/aspiration at any flow rate, participants reported subjective complaints of increased difficulty swallowing at higher flow rates [35]. In another study with healthy volunteers, a flow rate $>40 \mathrm{~L} / \mathrm{min}$ was associated with decreased swallowing function [36]. In healthy volunteers, high-flow nasal cannula was associated with enhanced swallowing function through reduced latency of the swallowing reflex [37]. In a study of healthy adults, flow rate effected the oral phase of swallowing via reduced lip closure, tongue control, and increase in oral residue; however, no statistically significant effect was observed on pharyngeal stage of swallowing [38].

In a retrospective study examining oral alimentation initiation in a patient population using high-flow nasal cannula, cognition and ambulatory status were significant factors for diet tolerance. All participants in the study were able to initiate an oral diet; however, $80 \%(8 / 10)$ required diet modification, $87.5 \%$ (7/8) required modification or exclusion of liquid consistency, and 50\% (5/10) exhibited silent penetration or aspiration during modified barium swallow study. Of note, 75\% exhibited tolerance of diet 1-week post-initiation and were able to continue oral intake and maintain respiratory and medical stability. Patients in this study tolerating the most liberal or least restrictive diets were cognitively intact and ambulatory independently or with supervision [39•]. In a prospective study that included 50 adult critically ill patients, a pathway was applied to patients on high-flow nasal cannula to help with the decision to initiate oral intake. This pathway consisted of an assessment of adequacy of respiratory status, an assessment of adequacy of mental status, and passing the Yale Swallow Protocol, which is a swallowing screening tool (see Table 3). Patients with adequate respiratory and mental state, but who did not pass the swallow screen, underwent a fiber optic evaluation of swallowing to assess if an oral diet modification would be applicable. Thirty-four (68\%) patients passed the initial swallow screen and were successfully initiated on oral feeding. Five (10\%) patients did not pass the initial swallow screen and were subsequently found to have pharyngeal phase dysphagia. These patients received a modified oral diet. Eleven (22\%) patients had severe respiratory illness and were thus kept nil per os [22].

The physiological studies had small sample size and were mostly performed in healthy adults. In aggregate, they indicate the feasibility of oral diet in healthy adults on high-flow nasal cannula. Limited clinical data in critically patients point to the same direction. In the latter patients, the neurological and functional statuses are important determinants as to whether they can tolerate oral diet.

\section{Nutrition Support via Enteral Route in Patients with High-Flow Nasal Cannula}

In an observational study of 40 patients on high-flow nasal cannula in the ICU, 21 (52\%) received enteral nutrition. All patients were underfed, but paradoxically those on enteral nutrition received less calories ( $365 \mathrm{kcal} / \mathrm{day}$ vs $600 \mathrm{kcal} / \mathrm{day}$ ) and proteins ( $18.5 \mathrm{~g} /$ day vs $22 \mathrm{~g} /$ day) compared with patients on oral diet. Patients who received enteral nutrition had a longer length of stay compared with patients who received oral nutrition ( 14 day vs $4 \mathrm{~d}$; $P<0.03$ ). Patients on enteral nutrition also had a numerically higher rate of intubation and deaths, but there was no statistically significant difference. The study was small and likely not powered to detect differences in these outcomes [23•]. Importantly, patients who received enteral nutrition had higher APACHE II and SOFA scores as compared with those who received oral diet. The higher baseline illness severity of these patients could have explained the choice of enteral nutrition as the route for feeding, and their worse outcomes.

Another study reported on a patient who was on mechanical ventilation and, after extubation, on high-flow nasal cannula. The patient received enteral nutrition with a feeding tube equipped with multichannel bioimpedance sensors capable of detecting and quantifying reflux events. The change of invasive mechanical ventilation to high-flow nasal cannula was associated with a marked increase in the number and magnitude of reflux events. The authors of the study hypothesized that the high flow of air leads to dilatation and increase in pressure of the stomach during inspiration and a precipitous drop of pressure during exhalation with consequent
Table 3 Components of the Yale swallow protocol
Assessment of exclusion criteria (e.g. nil per os order by the physician or head-of-bed restrictions to $<30^{\circ}$ )
Cognitive (three orientation questions)
Oral-mechanism examination (labial closure, lingual range of motion, and facial symmetry)
3-oz water challenge performed in a upright position (assess for interrupted drinking, cough, or choking)
Pass: ability to drink $3 \mathrm{oz}$ of water in uninterrupted way and without signs of aspiration
Fail: Interrupted drinking of $3 \mathrm{oz}$ of water, or signs of aspiration such as coughing or choking

Reference for the protocol: study by Suiter et al. [49] 
reflux [40]. This study raises concern for the safety of enteral nutrition in patients on high-flow nasal cannula although this was a single-patient study. It is unclear if the same pattern of aspiration is consistently observed in these patients.

\section{Nutrition via Oral Route in Patients on Non-invasive Ventilation}

Patients in acute respiratory failure are typically reassessed both clinically and with blood gas $1-2 \mathrm{~h}$ after initiation of non-invasive ventilation. Another clinical reassessment is typically performed after $4 \mathrm{~h}$ of initiation of non-invasive ventilation. At that point, if the patient is feeling better, a small break from non-invasive ventilation can be taken and oral swallowing can be assessed at bedside. If the patient continues to improve in the respiratory failure, periodic small breaks can then be scheduled in which the patient can receive oral nutrition.

A generally accepted rule is that patients on non-invasive ventilation do not receive oral diet during the ventilation period. This notion was challenged by a study of 15 patients with COPD exacerbation. These patients wore a nasal mask and were ventilated with non-invasive bilevel positive pressure ventilation. Water boluses of 5 and $10 \mathrm{ml}$ were provided to the patients, and the swallowing performance and breathing-swallowing interactions were evaluated. Noninvasive ventilation was associated with improved efficiency and dyspnea scores, but is also led increased autotriggering, which is a type patient-ventilator asynchrony. The addition of a device that allowed an off-switch during swallowing eliminated autotriggering [41]. It has been suggested that the improved swallowing with non-invasive ventilation could be due to an increase in the operative lung volume, increase in subglottic pressure leading to less aspiration, unloading of the respiratory muscles, and decrease in carbon dioxide tension [42]. While provocative, this study was small and the findings should be viewed as preliminary.

\section{Nutrition Support via Enteral Route in Patients on Non-invasive Ventilation}

An alternative for patients on non-invasive ventilation who cannot tolerate oral feeding is the insertion of a nasoenteral tube for feeding. However, there is concern for aspiration, and the feeding tube can lead to air leakage. Measures to mitigate air leakage include padding of the tube and skin with silicone dressing and the use of small caliber tubes [43]. If available, an adapter for the feeding tube can be added, or a mask with an incorporated adapter can be applied [43].

In a study of 107 patients on non-invasive ventilation for $>48$ h, $60(56 \%)$ received enteral nutrition. Patients on enteral nutrition had significantly more airway complications (53\% vs $32 \%, P=0.03$ ), defined as episodes of vomiting leading to desaturation, mucus plug, and aspiration pneumonia, as compared with patients not on enteral feeding. After multivariate adjustment, enteral feeding remained a significant risk factor for airway complications (odds ratio 2.46, 95\% CI 1.03-6.13). In-hospital mortality was numerically higher in patients on enteral feeding, but there was no statistically significant difference [44]. The sample size was, however, small and likely not powered for that outcome. A limitation of the study was that no adjustment for severity of illness was performed in the multivariate analysis. Thus, there was no accounting for an important confounding factor. In another study of 1075 patients admitted to the ICU and who required non-invasive ventilation for more than 2 consecutive days, the implementation of enteral nutrition was associated with a two-fold increase in the risk of 28-day mortality, two-fold increase in the risk of invasive mechanical ventilation, and fewer ventilator-free days [21].

\section{Proposed Management}

Factors that can impede adequate nutrition in non-intubated patients in acute respiratory failure include inability to eat due to non-invasive ventilation, mental status, increased energy consumption from underlying illness, and intolerance resulting in nausea or vomiting. Loss of appetite is a common cause for reduced oral intake in acutely ill patients. In a study that included 750 hospitalized patients, the most common patient related reason for insufficient dietary intake was poor appetite [45]. Improving food taste, inclusion of a patient self-menu selection, flexible meal serving time, and adequate cooking time are ways of circumventing the loss of appetite of these patients [46]. Improving the visual presentation of the food is an overlooked yet low-cost strategy that can lead to increased food intake [47]. Intake of oral nutritional supplements can lead to an increase protein intake [46]. Individualized nutritional care, including a detailed nutritional assessment and provision of fortified meals and nutritional supplements, increases energy and protein intake in malnourished in-hospital patients [48]. Tackling organizational factors, such as the lack of systematic screening for nutritional risk, and improving nutrition education are important as these are often major determinants of adequate nutrition [45].

The decision to initiate nutrition in a non-intubated patient in acute respiratory failure should consider the patient's nutritional risk, the severity of the acute respiratory failure, the mental status, and the chosen method of oxygen delivery (high-flow nasal cannula vs non-invasive ventilation). The most immediate factor to consider is the severity of acute respiratory failure and potential need for endotracheal intubation. For instance, if the need for endotracheal intubation is imminent, the patient should be kept nil per os. Similarly, if there is a reasonable probability that the patient may require 
endotracheal intubation within the next $12 \mathrm{~h}$, it is prudent to initially keep the patient nil per os so as to minimize the risk of aspiration during the procedure.

The mental status and level of alertness are also important. Patients with hypoxic respiratory failure not uncommonly become agitated whereas those with hypercapnic respiratory failure tend to be drowsy. The level of alertness usually improves with treatment, and this is most evident in patients with hypercapnic respiratory failure treated with non-invasive ventilation. In a patient with declined mental status in whom treatment has just started, it is prudent to wait a few hours before assessing the patient for swallowing.

The expected duration of acute respiratory failure should also be factored in. For instance, postponing oral intake for a few hours may be warranted in a patient in frank respiratory failure secondary to cardiogenic pulmonary edema, a condition that tends to improve rapidly with treatment. On the other hand, in a patient with acute respiratory failure secondary to pneumonia, a rapid improvement is unlikely, and early initiation of feeding may be warranted.

We recommend a multidisciplinary and protocolized approach to the initial nutrition management of these patients, similar to the protocol in the study by Leder et al. [22]. An initial assessment by a multidisciplinary team should focus on whether it is safe to initiate oral diet. This bedside assessment should include mental status evaluation, the Yale swallowing screening protocol [49], and an assessment of severity of illness.

The goals of swallow screening are to determine the likelihood that dysphagia is present, ascertain aspiration risk,

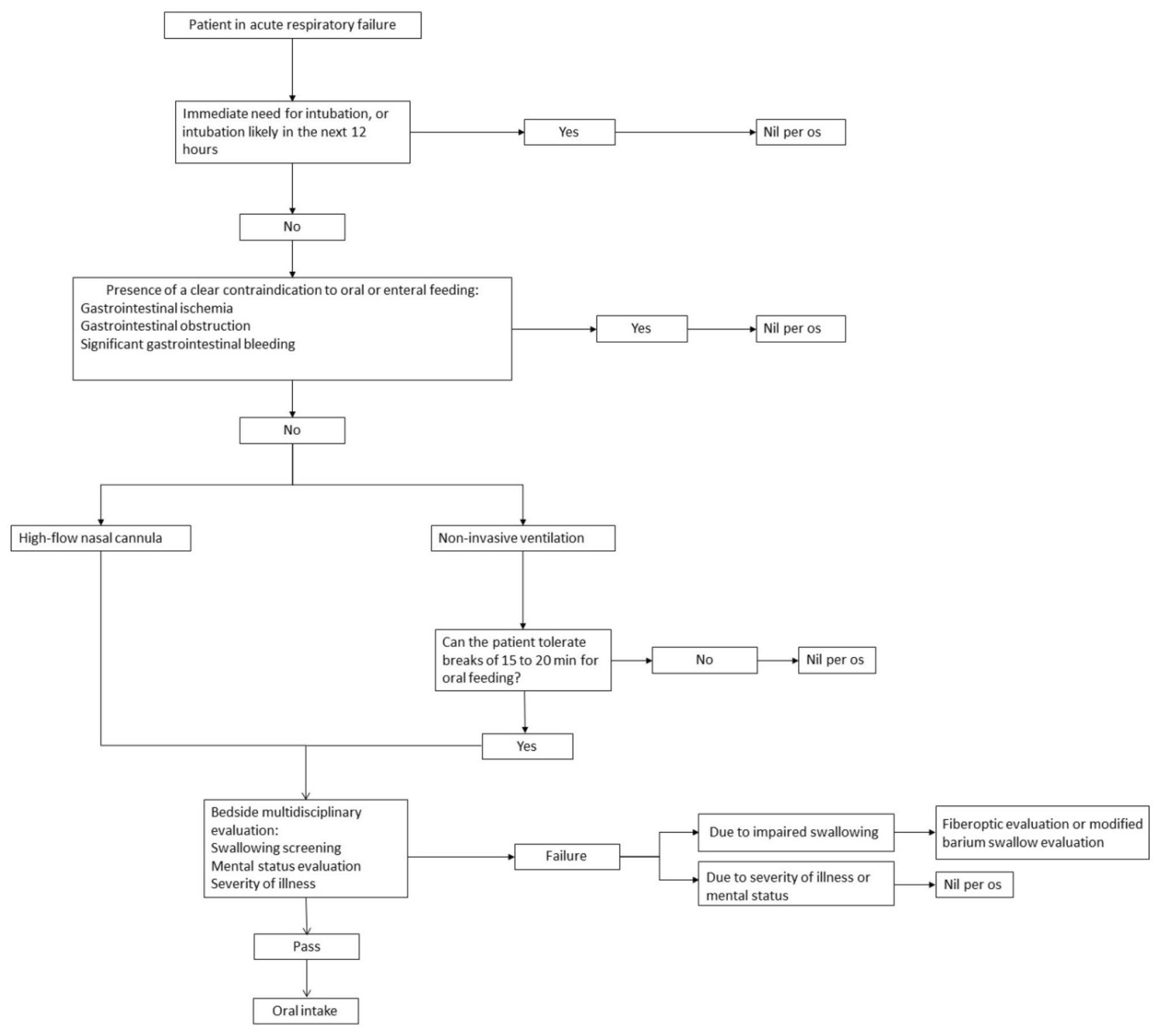

Fig. 1 Early nutritional approach to patients in acute respiratory failure on high-flow nasal cannula or non-invasive positive pressure ventilation 
determine need for formal swallow evaluation, and determine when it is safe to resume an oral diet. The Yale swallow screening protocol is a valid and reliable tool for discerning aspiration risk. In one study that used videofluoroscopic swallow study as the reference standard, the Yale protocol had a negative predictive value of $100 \%$, which means none of the patients who passed the screening aspirated during the videofluoroscopic swallow study. The positive predictive value was $78 \%$, implying that some patients failed the Yale swallow screening protocol but subsequently passed the videofluoroscopic swallow study. To implement the Yale swallow screening protocol, a trained clinician simply performs a brief cognitive assessment, oral mechanism exam, and administers a three-ounce water trial. Cognitive assessment consists of basic orientation questions targeting person, place, and time. The oral mechanism exam consists of assessing labial closure, lingual strength and range of motion, and facial symmetry [49]. The three-ounce water trial has a pass/fail criteria and is conducted to assess for overt signs and symptoms of aspiration, i.e., coughing and throat clearing. The clinician instructs the patient to drink the entire three ounces of water via slow and steady sequential drinks. The patient must consume the entire three ounces uninterrupted with no signs/ symptoms of aspiration to meet the pass criteria (see Table 3) $[49,50]$.

If deemed safe, patient should be started on oral diet within the first $24 \mathrm{~h}$. If not, then the reason for not initiating oral diet should dictate the next step (see Fig. 1). For instance, if severity of illness or mental status are the driving factors, the patient is kept nil per os and frequent reassessments should be performed as the patient's condition may change. If failure of the swallow screening is the main factor, further assessment with either fiberoptic evaluation or modified barium swallow test should be performed. In these patients, whose respiratory status is tenuous, fiberoptic evaluation is favored over modified barium swallow test since it can be performed at bedside.

We suggest the placement of a small-bore feeding tube if the patient is unable to receive oral diet or at least $50 \%$ of the caloric goal via oral intake after $48 \mathrm{~h}$ in the absence of contraindication to the gastrointestinal tract use (e.g. bowel obstruction). This is in line with a BAPEN statement, which recommends the consideration of nasogastric feeding in patients with Covid-19 in the setting of inadequate oral intake after 2 days [51]. There are two caveats, however, to the use of enteral nutrition in these patients. First, studies showed worse outcomes with the use of enteral nutrition in this patient population [23•, 24], but the studies were observational and susceptible to confounding. Second, the process of placing a feeding tube is not without risks and can be particularly dangerous in this patient population whose respiratory status is tenuous. For instance, in a review of 1822 tubes placed in 729 patients, misplacement of a feeding tube into the respiratory tree occurred in 23 (3.2\%) patients. Nine (1.2\%) patients developed a pneumothorax; of these, 4 patients died [52]. The use of electromagnetic device to guide placement of feeding tube has the potential to minimize tube misplacement [53]. Endoscopic placement is also an option, but there may be logistic barriers to the routine use of endoscopic placement [54]. If the placement is blind, the development of cough or respiratory distress should lead to abortion of the procedure. No further blind attempts should be performed as multiple attempts have been described in patients who develop complications.

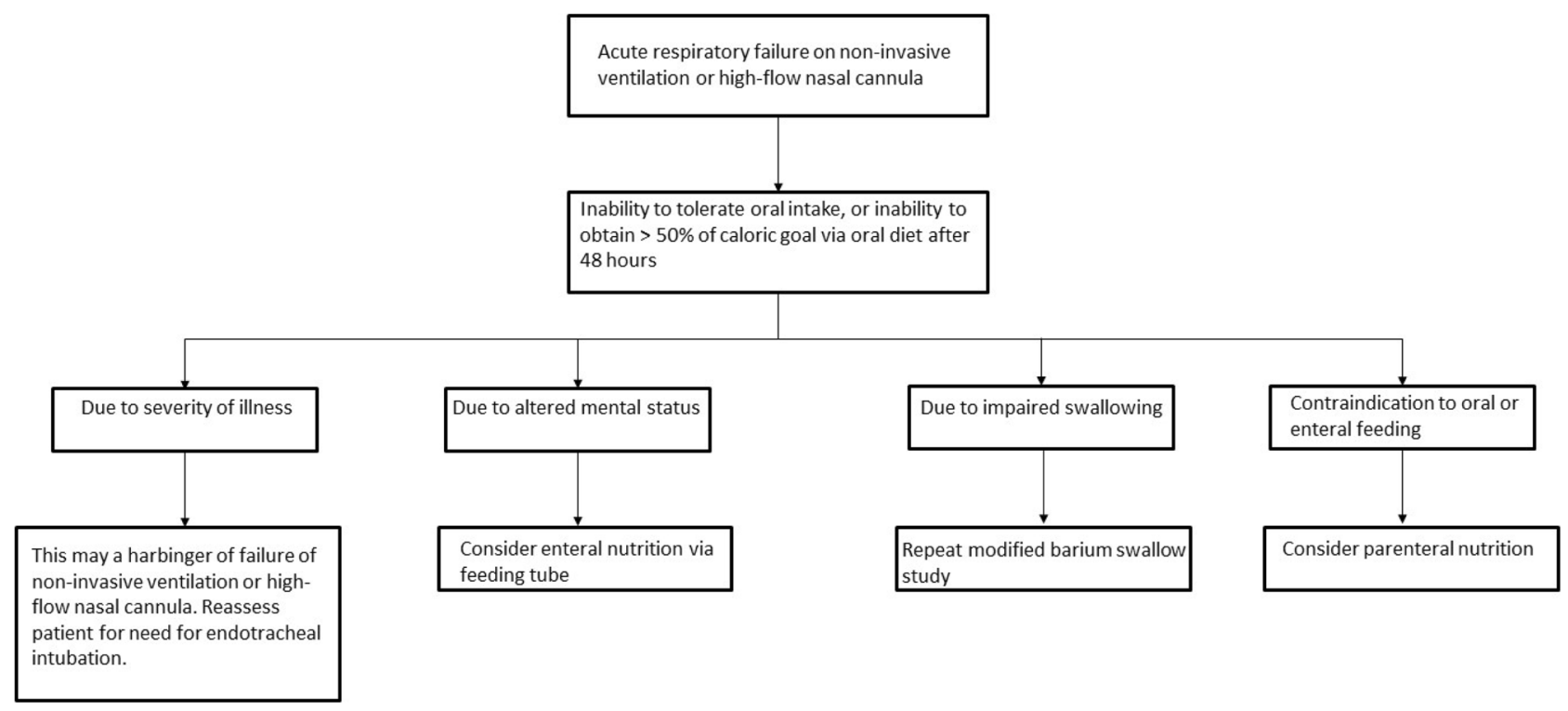

Fig. 2 Approach to the patient in acute respiratory failure on high-flow nasal cannula or non-invasive positive pressure ventilation after $48 \mathrm{~h}$ 
That a patient cannot initiate oral diet due to severity of illness after $48 \mathrm{~h}$, particularly in the presence of respiratory distress, may be a sign of failure of either non-invasive ventilation or high-flow nasal cannula and should prompt consideration for endotracheal intubation and invasive mechanical ventilation with subsequent initiation of enteral nutrition. Finally, if after $48 \mathrm{~h}$ patient is not receiving oral or enteral diet because of contraindication to the use of the gastrointestinal tract, parenteral nutrition should be considered (see Fig. 2).

\section{Conclusion}

The nutrition management of patients with acute respiratory failure on non-invasive ventilation or high-flow nasal cannula requires a truly multidisciplinary approach. Clinical judgment plays an important role in deciding for instance if the patient is stable to tolerate oral intake or if endotracheal intubation is imminent. However, decisions regarding nutrition should be informed by well tested clinical pathways. It is critical to consider overall respiratory status, type of respiratory failure, cognitive status, nutritional risk, and swallowing status. The Yale swallow protocol is an important tool that helps guide decisions and should be integrated in the assessment of these patients. Early involvement of the multidisciplinary team members, including nurse, respiratory therapist, dietitian, speech pathologist, and physician, is paramount as these patients present unique challenges that require the expertise of different specialties. To improve patient care, we believe this an area with ample opportunity for clinical research, ranging from implementation research, to development and testing of protocols and clinical pathways, and to clinical trials.

\section{Declarations}

Conflict of interest The authors do not have any potential conflicts of interest to disclose.

Human and Animal Rights and Informed Consent This article does not contain any studies with human or animal subjects performed by any of the authors.

\section{References}

\section{Papers of particular interest, published recently, have been highlighted as:}

\section{- Of importance}

1. National Heart L, et al. Initial trophic vs full enteral feeding in patients with acute lung injury: the EDEN randomized trial. JAMA. 2012;307(8):795-803.
2. Nguyen NQ, et al. The impact of delaying enteral feeding on gastric emptying, plasma cholecystokinin, and peptide YY concentrations in critically ill patients. Crit Care Med. 2008;36(5):1469-74.

3. Roussos C, Koutsoukou A. Respiratory failure. Eur Respir J. 2003;22(47 suppl):3s-14s.

4. Neder JA, Berton DC, O'Donnell DE. Arterial blood gases in the differential diagnosis of hypoxemia. $\mathrm{J}$ Bras Pneumol. 2020;46(5): p. e20200505.

5. Gunning KEJ. Pathophysiology of respiratory failure and indications for respiratory support. Surg Infect (Larchmt). 2003;21(3):72-6.

6. Sarkar M, Niranjan N, Banyal PK. Mechanisms of hypoxemia. Lung India : official organ of Indian Chest Society. 2017;34(1):47-60.

7. Meduri GU, Conoscenti CC, Menashe P. Noninvasive mechanical ventilation in acute respiratory failure: happy 30-year anniversary! Chest. 2020;157(2):255-7.

8. Meduri GU, et al. Noninvasive Face Mask Ventilation in Patients with Acute Respiratory Failure. Chest. 1989;95(4):865-70.

9. Osadnik, CR et al. Non-invasive ventilation for the management of acute hypercapnic respiratory failure due to exacerbation of chronic obstructive pulmonary disease. Cochrane Database Syst Rev. 2017;7(7):Cd004104.

10. Berbenetz N, et al. Non-invasive positive pressure ventilation (CPAP or bilevel NPPV) for cardiogenic pulmonary oedema. Cochrane Database Syst Rev. 2019;4(4):Cd005351.

11. Papazian L, et al. Use of high-flow nasal cannula oxygenation in ICU adults: a narrative review. Intensive Care Med. 2016;42(9):1336-49.

12. Voigt E, Pelikan J. CO2-measurement during ventilation. Drägerwerk $\mathrm{AG} \& \mathrm{Co} . \mathrm{KGaA}$.

13. Gupta RD, et al. Indirect Calorimetry: From Bench to Bedside. Indian journal of endocrinology and metabolism. 2017;21(4):594-9.

14. Wischmeyer PE, Molinger J, Haines K. Point-counterpoint: indirect calorimetry is essential for optimal nutrition therapy in the intensive care unit. Nutr Clin Pract. 2021;36(2):275-81.

15. McClave SA, et al. Guidelines for the provision and assessment of nutrition support therapy in the adult critically ill patient: Society of Critical Care Medicine (SCCM) and American Society for Parenteral and Enteral Nutrition (A.S.P.E.N.). JPEN J Parenter Enteral Nutr. 2016;40(2):159-211.

16. McClave SA, Omer E. Point-counterpoint: indirect calorimetry is not necessary for optimal nutrition therapy in critical illness. Nutr Clin Pract. 2021;36(2):268-74.

17. Vermeeren MA, Schols AM, Wouters EF. Effects of an acute exacerbation on nutritional and metabolic profile of patients with COPD. Eur Respir J. 1997;10(10):2264-9.

18.• Whittle J, et al. Persistent hypermetabolism and longitudinal energy expenditure in critically ill patients with COVID-19. Critical Care. 2020;24(1):581. This study illustrates the presence of a hyper-metabolic status in patients with COVID-19. It also demonstrated the hypermetabolism exceedes Harris-Bennedict equation prediction after the first week. This hypermetaboism increases throughout the hospitalization with a peak in the third week.

19. Martindale R, et al. Nutrition therapy in critically ill patients with coronavirus disease 2019. JPEN. J Parenter Enter Nutr. 2020;44(7):1174-1184.

20. Reeves A, et al. Energy and protein intakes of hospitalised patients with acute respiratory failure receiving non-invasive ventilation. Clin Nutr. 2014;33(6):1068-73.

21. Terzi N, et al. Initial nutritional management during noninvasive ventilation and outcomes: a retrospective cohort study. Critical care (London, England). 2017;21(1):293-293.

22. Leder SB, et al. Oral alimentation in neonatal and adult populations requiring high-flow oxygen via nasal cannula. Dysphagia. 2016;31(2):154-9. 
23. Zerbib O, et al. Energy and protein intake in critically ill people with respiratory failure treated by high-flow nasal-cannula oxygenation: an observational study. Nutrition. 2021;84:111117. Observational study looking at energy and protein intake for patients on high-flow nasal cannula for management of acute respiratory failure. This study showed that patients on highflow nasal cannula were significantly underfed.

24. Rice TW, et al. Initial trophic vs full enteral feeding in patients with acute lung injury: the EDEN randomized trial. JAMA. 2012;307(8):795-803.

25. Arabi YM, et al. Permissive underfeeding or standard enteral feeding in critically ill adults. N Engl J Med. 2015;372(25):2398-408.

26. Borek P, et al. Analysis of outcomes of the NRS 2002 in patients hospitalized in nephrology wards. Nutrients. 2017;9(3)

27. Norman K, et al. Prognostic impact of disease-related malnutrition. Clin Nutr. 2008;27(1):5-15.

28. White JV, et al. Consensus statement: Academy of Nutrition and Dietetics and American Society for Parenteral and Enteral Nutrition: characteristics recommended for the identification and documentation of adult malnutrition (undernutrition). JPEN J Parenter Enteral Nutr. 2012;36(3):275-83.

29. Kondrup J, et al. ESPEN guidelines for nutrition screening 2002. Clin Nutr. 2003;22(4):415-21.

30. Heyland DK, et al. Identifying critically ill patients who benefit the most from nutrition therapy: the development and initial validation of a novel risk assessment tool. Critical care (London, England). 2011;15(6):R268-R268.

31. Kondrup J, et al. Nutritional risk screening (NRS 2002): a new method based on an analysis of controlled clinical trials. Clin Nutr. 2003;22(3):321-36.

32. Ferguson M, et al. Development of a valid and reliable malnutrition screening tool for adult acute hospital patients. Nutrition. 1999;15(6):458-64.

33. Evans DC, et al. The use of visceral proteins as nutrition markers: an ASPEN position paper. Nutr Clin Pract. 2021;36(1):22-8.

34. Wojda TR, et al. Ultrasound and computed tomography imaging technologies for nutrition assessment in surgical and critical care patient populations. Current Surgery Reports. 2015;3(8):21.

35. Allen K, Galek K. The influence of airflow via high-flow nasal cannula on duration of laryngeal vestibule closure. Dysphagia. 2020;1-7.

36. Oomagari $\mathrm{M}$, et al. Swallowing function during high-flow nasal cannula therapy. 2015, Eur Respiratory Soc.

37. Sanuki T, et al. Effect of nasal high-flow oxygen therapy on the swallowing reflex: an in vivo volunteer study. Clin Oral Invest. 2017;21(3):915-20.

38. Eng K, et al. Evaluation of swallow function on healthy adults while using high-flow nasal cannula. Perspectives of the ASHA Special Interest Groups. 2019;4(6):1516-24.

39. Flores MJ, et al. Initiation of oral intake in patients using high-flow nasal cannula: a retrospective analysis. Perspectives of the ASHA
Special Interest Groups. 2019;4(3):522-531. This is a retrospective analysis of initiating diet in patients requiring high-flow nasal cannula. It illustrated that all the critically ill patients in the study were able to start an oral diet and most tolerated the diet 1 week after initiation while maintaining respiratory and medical stability.

40. Kagan I, et al. Reflux events detected by multichannel bioimpedance smart feeding tube during high flow nasal cannula oxygen therapy and enteral feeding: first case report. J Crit Care. 2020;60:226-9.

41. Terzi N, et al. Noninvasive ventilation and breathing-swallowing interplay in chronic obstructive pulmonary disease*. Crit Care Med. 2014;42(3):565-73.

42. Shaikh H, Laghi F. Eating on noninvasive ventilation*. Crit Care Med. 2014;42(3):737-8.

43. Brill A-K. How to avoid interface problems in acute noninvasive ventilation. Breathe. 2014;10(3):230-42.

44. Kogo M, et al. Enteral nutrition is a risk factor for airway complications in subjects undergoing noninvasive ventilation for acute respiratory failure. Respir Care. 2017;62(4):459-67.

45. Kondrup J, et al. Incidence of nutritional risk and causes of inadequate nutritional care in hospitals. Clin Nutr. 2002;21(6):461-8.

46. Thibault R, et al. Assessment of food intake in hospitalised patients: a 10-year comparative study of a prospective hospital survey. Clin Nutr. 2011;30(3):289-96.

47. Spence C. Hospital food Flavour. 2017;6(1):3.

48. Starke J, et al. Short-term individual nutritional care as part of routine clinical setting improves outcome and quality of life in malnourished medical patients. Clin Nutr. 2011;30(2):194-201.

49. Suiter DM, Sloggy J, Leder SB. Validation of the Yale Swallow Protocol: a prospective double-blinded videofluoroscopic study. Dysphagia. 2014;29(2):199-203.

50. Suiter DM, Leder SB. Clinical utility of the 3-ounce water swallow test. Dysphagia. 2008;23(3):244-50.

51. BAPEN. Route of nutrition support in patients requiring NIV \& CPAP during the COVID-19 response. 2020, April 21 [cited 2021 May 16]; Available from: https://www.bapen.org.uk/resources-andeducation/education-and-guidance/covid-19.

52. de Aguilar-Nascimento JE, Kudsk KA. Clinical costs of feeding tube placement. JPEN J Parenter Enteral Nutr. 2007;31(4):269-73.

53. Powers J, et al. Improved safety and efficacy of small-bore feeding tube confirmation using an electromagnetic placement device. Nutr Clin Pract. 2018;33(2):268-73.

54. Gerritsen A, et al. Electromagnetic guided bedside or endoscopic placement of nasoenteral feeding tubes in surgical patients (CORE trial): study protocol for a randomized controlled trial. Trials. 2015;16:119.

Publisher's Note Springer Nature remains neutral with regard to jurisdictional claims in published maps and institutional affiliations. 